\title{
Health Related Quality of Life of Patients with Vertebral Bone Metastasis after Palliative Surgery
}

\author{
Cristiane Thomaz de Aquino Exel Andrade ${ }^{1}$, Juliana Maria de Paula ${ }^{2}$, Namie \\ Okino Sawada ${ }^{3}$ \\ ${ }^{1}$ (Physiotherapist. Oncological Institute of Ribeirão Preto. Ribeirão Preto, São Paulo, Brazil) \\ ${ }^{2}$ (Registered Nurse. University of Sao Paulo. Ribeirão Preto, São Paulo, Brazil) \\ ${ }_{3}^{3}$ (Registered Nurse. University of Sao Paulo. Ribeirão Preto, São Paulo, Brazil)
}

\begin{abstract}
Aim: To evaluate the impact of spinal decompression surgery on the Health Related Quality of Life of patients affected by metastasis at some level of the spine. Method: This was a quantitative, descriptive, correlational and longitudinal, prospective study which included four evaluations: before surgery (T0), two (T1), four (T2) and six (T3) months after surgery. The instruments used for data collection were: structured questionnaire for sociodemographic and clinical characterization of the sample; Revised Faces Pain Scale (FPS-R); Health Related Quality of Life evaluation questionnaire (EORTC QLQ-C30). Results: The sample consisted of 22 patients, with the most prevalent primary tumors being breast, prostate, lung and colorectal. In the pre-operative period (TO), the participants presented low scores for Global Health Status/Quality of Life and for almost all domains of the Functional Scale and Symptom Scale. In the three moments (T1, T2 and T3), there was an improvement in the role functioning, pain, insomnia and constipation, although there was no significant difference in activities of daily living at any of the three moments analyzed after surgery. Conclusion: There was improvement in health related quality of life.
\end{abstract}

Keywords: Palliative Surgery, Vertebral Metastasis, Neoplasms, Quality of Life

\section{Introduction}

The cancer cell may send its primary site to several organs (liver, lungs, brain, bones) via the blood or lymphatic circulation and thus constituting so-called metastasis. The most affected organs are the lungs, liver and bones. Considering only bone metastasis, the tumors that metastasize more to this organ are prostate, breast, kidney, lung and thyroid [1]. The highest incidence of metastatic bone lesions occur in the axial skeleton. The spine is the most common site, with prevalence rates ranging from $30 \%$ to $80 \%$ [2]. Vertebral metastasis affects $20 \%$ to $50 \%$ of patients with cancer and represent an abrupt change in the quality of life of individuals due the functional impact that it generates [3]. Among the individuals who develop vertebral metastasis during the course of the cancer, $10 \%$ present symptoms or sequelae [4]. One of the most prevalent complaints being spinal compression, considered an oncologic emergency that can compromise the quality of life of the patient [5]. This most frequently occurs in the thoracic spine (60\%), followed by lumbosacral spine (25\%) and cervical spine $(10 \%)$. These percentages are related to the relative distribution of blood flow and the presence of bone in each vertebral segment [6-7]. The vertebrae of the spine are the sites most affected by bone metastasis. The thoracic vertebrae are affected in $70 \%$ of cases, followed by lumbar vertebrae in $20 \%$ of cases and the cervical vertebrae in the remaining $10 \%$ [8]. There are many morbidities that bone metastasis can cause for the patients, such as: pain that is difficult to control, sensory and motor deficits, dependence on caregivers to perform basic activities of daily living and pathological fractures that need surgery. Studies show that $9 \%$ to $29 \%$ of cases present a pathological fracture, and $90 \%$ of these cases will have to undergo surgery to correct the condition [9]. The prognosis of patients with bone metastasis is highly variable, being influenced by the location of the metastatic lesion, the histological subtype of the primary tumor and the presence of other metastases. Most often these patients have no more curative potential, with palliative treatment being indicated [10].

The last two decades has seen a revolution in oncological therapy with new chemotherapeutic drugs, the development of immunotherapy for certain tumors, new radiotherapy techniques with fewer side effects and greater response rate. Thus, there has been an increase in life expectancy and a higher rate of late recurrences. The management of these recurrences has also undergone changes in recent years. The improvement of surgical techniques for bone metastasis in the spine allows better recovery of patients with satisfactory resolution of the neurological symptoms presented by them [11]. With a higher success rate in the various current treatments, a critical and detailed assessment of the quality of life of these patients following treatment is also necessary. Especially considering patients with spinal metastasis, this analysis is extremely challenging because they are people with numerous other comorbidities, as well as presenting various pre-operative symptoms that deteriorated their quality of life [12]. 
This study was designed to evaluate the health related quality of life of patients undergoing palliative surgery for spinal metastasis in the orthopedics service of the Clinical Hospital of Ribeirão Preto Medical School - USP. The aim was to analyze the actual benefits of the surgical procedure indicated using previously validated instruments.

\section{Materials and methods}

This was a quantitative, descriptive, correlational and longitudinal, prospective study which included four evaluations: before the surgery, two, four and six months after the surgery. All patients with an indication of surgery for stabilization of cervical, thoracic or lumbar vertebras compromised by neoplastic cells, in the period from October 2013 to August 2014, referred to the Spinal Service clinic of the Department of Biomechanics, Medicine and Rehabilitation of the Locomotor System of the Clinical Hospital of the Ribeirão Preto Medical School, University of São Paulo (HC-FMRP), were eligible for this study. Exclusion criteria for the study were: primary tumors of the spine, severe depression diagnosis reported in the medical records that would hinder the understanding of the questionnaires to be answered, and any cognitive impairment that would prevent the patient responding adequately to the study questionnaires.

Data were collected through interviews conducted by the researcher, in the inpatient units (preoperatively) and in the Spinal Service clinic of the Department of Biomechanics, Medicine and Rehabilitation of the Locomotor System of HC-FMRP (at two, four and six months after the date of surgery). Interviews were conducted at four different times for each patient, namely: before surgery (T0) in the inpatient units of the HCFMRP Spinal Service because the patients were hospitalized for control of pain and symptoms and pre-operative diagnostic examinations, and at two (T1), four (T2) and six (T3) months after surgery at the clinic of the same service.

The questionnaires were composed of four subdivisions: socio-demographic and clinical (description of gender, age, schooling, occupation and professional situation, family income, type of tumor, metastasis location, type of surgical procedure); evaluation of the pain through the Revised Face Pain Scale (FPS-R) (13), which is a self-reported scale with six horizontally presented faces, with expressions of crying or smiling to express different degrees of pain from "without pain" to "the greatest pain possible", making the metric correlation of 010, where zero corresponds to no pain and ten to the greatest pain possible; evaluation of the quality of life EORTC QLQ-C30 (European Organisation for Research and Treatment of Cancer Questionnaire "core" 30 items) (14-15) developed by the European Organisation for Research and Treatment of Cancer with the aim of evaluating the Quality of Life of patients with lung cancer, and from this study this scale was universally recognized as valid and reliable to evaluate Quality of Life in a population with cancer; evaluation of functional capacity through the Barthel Index (16), with this instrument belonging to the field of evaluation of Activities of Daily Living (ADLs) and measuring the functional independence related to the personal care, mobility, locomotion and physiological habits.

Statistical analysis was performed using the Statistical Package for the Social Sciences (SPSS) version 16.0. Descriptive analyzes of simple frequency were performed for the categorical variables (gender, race, marital status, education level, occupation, employment status, monthly income, primary diagnosis, type of surgery, level of metastatic lesion and post-operative treatment) and of central tendency (median and mean) and variability (standard deviation - SD) for the continuous variables of age and measures of HRQoL. To compare the scores that evaluate the HRQoL and functional capacity presented at the pre-operative moment (T0) and the results perceived by the patients at two (T1), four (T2) and six (T3) months after surgery, Student's paired t-test was used. To compare the measures of the HRQoL domains, pain (FPS-R scale) and functional limitation perceived before and after the surgery, Student's paired t-test was used. All statistical tests were performed considering a 0.05 significance level. This study was approved by the Research Ethics Committee of the University of São Paulo at Ribeirão Preto College of Nursing. A consent form was signed by each patient prior to the start of the study.

\section{Results}

A total of 26 patients presented clinical spinal cord compression in the 11-month study period, undergoing corrective surgery at the Clinical Hospital of FMRP - USP. Of these, three patients were diagnosed with multiple myeloma and one patient diagnosed with primary tumor of spine, with these excluded from the final analysis, leaving a total of 22 participants. There were 11 female (50\%) and $11(50 \%)$ male patients. Ages ranged from 33 to 87 years with a median of 57.00 (standard deviation $(\mathrm{SD})=13.50$ ); with $45 \%$ of the patients in the age group over 60 years. Only two (9.1\%) patients had complete higher education, and $68.2 \%$ of the participants had only elementary level education. At the time the questionnaire was applied, at the pre-operative moment and at the three post-operative moments, none of the study participants were working, with $50 \%$ being retired. The mean monthly income of each of the participants was divided as follows: $13(59.1 \%)$ received one minimum wage, four $(18.2 \%)$ received two minimum wages, two $(9.1 \%)$ received up to three minimum wages 
and three $(13.6 \%)$ received four or more minimum wages. Table 1 summarizes the clinical characteristics of the sample. The most prevalent tumor was breast cancer, accounting for over $40 \%$ of the respondents. The posterior vertebral approach was the surgical approach most commonly used, and the lumbar and thoracic levels comprised almost $70 \%$ of the metastatic lesions. No further treatment was performed in $50 \%$ of the cases. Of the 22 patients included, 10 died during the study, with 17 participants remaining at the T1 moment, 14 at T2, and 12 at $\mathrm{T} 3$.

Table 1 - Frequency and percentage of the clinical characteristics of the patients with vertebral metastasis of the Clinical Hospital of Ribeirão Preto Medical School (HC-FMRP) Ribeirao Preto/SP - Brazil, 2013-2015 (n = 22).

\section{Clinical characteristics \\ Primary diagnosis (location of the cancer)}

$\begin{array}{lrr}\text { Breast } & 9 & 40.9 \\ \text { Prostate } & 4 & 18.2 \\ \text { Lung } & 3 & 13.6 \\ \text { Colon and rectum } & 3 & 13.6 \\ \text { Esophagus } & 1 & 4.5 \\ \text { Renal clear cells } & 1 & 4.5 \\ \text { Bladder } & 1 & 4.5\end{array}$

\section{Surgical procedure}

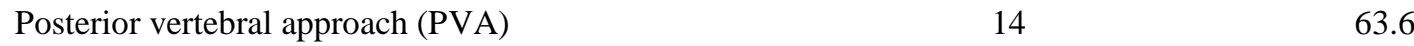

Kyphoplasty

Anterior vertebral approach (AVA)

$\mathrm{AVA}+\mathrm{PVA} *$

\section{Metastatic injury level}

Lumbar

Thoracic

Cervical

Thoracolumbar

Lumbosacral

Sacral

Cervicothoracic

\section{Postoperative treatment}

None

$11 \quad 50$

Radiotherapy

$5 \quad 22.7$

Chemotherapy

$4 \quad 18.2$

Chemotherapy + Radiotherapy

* Anterior vertebral approach plus posterior vertebral approach

\section{1- Health related quality of life (HROoL)}

The EORTC QLQ-C30 instrument (European Organization for Research and Treatment of Cancer Quality of Life Questionnaire "core" 30 item) was developed in order to evaluate the Quality of Life of cancer patients (Table 1) [15].

Figure 1 - Scores of the EORTC QLQ-C30 Version 3.0 (Adapted from Fayers et al., 2001)

\begin{tabular}{|c|c|c|c|}
\hline Scales & Domains & Questionnaire items & Item total \\
\hline SCALE OF GHS/QL * & & 29,30 & 2 \\
\hline \multirow{8}{*}{ FUNCTIONAL SCALE } & Physical functioning & $1,2,3,4,5$ & \multirow{3}{*}{$\begin{array}{l}5 \\
2 \\
4\end{array}$} \\
\hline & Role functioning & 6,7 & \\
\hline & Emotional functioning & $21,22,23,24$ & \\
\hline & Cognitive functioning & 20,25 & \multirow{2}{*}{$\begin{array}{l}2 \\
2\end{array}$} \\
\hline & Social functioning & 26,27 & \\
\hline & Fatigue & $10,12,18$ & \multirow{3}{*}{$\begin{array}{l}3 \\
2 \\
2\end{array}$} \\
\hline & Nausea and Vomiting & 14,15 & \\
\hline & Pain & 9,19 & \\
\hline
\end{tabular}


Health Related Quality of Life of Patients with Vertebral Bone Metastasis after Palliative Surgery

\begin{tabular}{|l|l|c|c|}
\hline \multirow{3}{*}{ SYMPTOMS SCALE } & Dyspnoea & 8 & 11 \\
\cline { 2 - 3 } & Insomnia & 13 & 12 \\
\cline { 2 - 4 } & Appetite Loss & 16 & 1 \\
\cline { 2 - 4 } & Constipation & 17 & 1 \\
\cline { 2 - 4 } & Diarrhea & 28 & 1 \\
\hline FINANCIAL DIFFICULTY SCALE & Financial difficulties & 1 \\
\hline
\end{tabular}

* Global Health Status/Quality of Life

Regarding the Global Health Status/Quality of Life (GHS/QoL) scale, in the pre-operative phase (T0) the patients presented a low score, with a mean of $46.97(\mathrm{SD}=28.12)$. After the surgery, a new analysis of the GHS/QoL showed a marked improvement after two months (T1), with a mean of 57.35 (SD $=25.15)$. There was maintenance of this improvement when analyzing the questionnaire at four (T2) (mean $=52.38 ; \mathrm{SD}=22.03$ ) and six months $(\mathrm{T} 3)$ (mean $=57.64 ; \mathrm{SD}=23.96)$.

Assessing the functional scale at the T0 moment, the means of four of the five variables were low, three of them below 50. Comparing the initial means of the pre-operative moment with the T1, T2 and T3 moments, for all domains there was a significant improvement, reflecting an improvement in the functional scale after surgery. The highest relative gains were achieved in the physical functioning and role functioning items, especially in the T3 period, with an improvement of $90 \%$ and $247 \%$ respectively.

Regarding the symptom scale, considering the eight variables, the greater benefit found after surgery was related to pain, which showed an improvement of 57.6\% at the T1 moment and approximately $46 \%$ at T2 and T3. There was significant improvement at six months (T3) in fatigue (26.3\%), nausea and vomiting (35.8\%), dyspnea (33.3\%), loss of appetite $(27.9 \%)$, insomnia $(40.7 \%)$, diarrhea $(54.1 \%)$ and constipation $(85.1 \%)$. There was no change in the comparison of the financial difficulty scale before and after surgery.

Using Student's t-test to compare paired variables of the pre-operative moment (T0) in relation to the $\mathrm{T} 1, \mathrm{~T} 2$ and T3 moments, role functioning, pain, insomnia and constipation showed statistically significant results $(\mathrm{p}<0.05)$ in the three analyzed moments.

\section{2 - Pain}

Table 2 presents the results of the revised Faces Pain Scale (FPS-R). At the pre-operative moment (T0), $72.8 \%$ of the patients presented a score equal to or greater than six for pain, with $31.9 \%$ scoring eight or ten. After the surgery, no patient presented pain greater than six, and at T1 approximately $30 \%$ were completely asymptomatic. At T3, the percentage of asymptomatic patients reached $25 \%$ and nine of the twelve patients still alive presented a maximum pain score of two. This analysis confirms what was seen in the EORTC QLQ-C30 questionnaire considering this symptom.

Table 2 - Frequency and percentage of the Revised Faces Pain Scale (FPS-R) of the patients with vertebral metastasis of the Clinical Hospital of Ribeirão Preto Medical School (HC-FMRP) Ribeirao Preto/SP - Brazil, 2013-2015 ( $\mathrm{n}=22)$.

\begin{tabular}{|c|c|c|c|c|}
\hline Revised Faces Pain Scale (FPS-R) & $T 0-n(\%)$ & $T 1-n(\%)$ & $T 2-n(\%)$ & $T 3-n(\%)$ \\
\hline 0 & $0(0)$ & $5(29.4)$ & $2(14.3)$ & $3(25.0)$ \\
\hline 2 & $3(13.6)$ & $2(11.8)$ & $7(50.0)$ & $6(50.0)$ \\
\hline 4 & $3(13.6)$ & $5(29.4)$ & $1(7.1)$ & $2(16.7)$ \\
\hline 6 & $9(40.9)$ & $5(29.4)$ & $4(28.6)$ & $1(8.3)$ \\
\hline 8 & $3(13.6)$ & $0(0)$ & $0(0)$ & $0(0)$ \\
\hline 10 & $4(18.3)$ & $0(0)$ & $0(0)$ & $0(0)$ \\
\hline Total & $22(100)$ & $17(100)$ & $14(100)$ & $12(100)$ \\
\hline
\end{tabular}

Table 3 shows the means, medians and standard deviations of the pain item of the EORTC QLQ C-30 instrument, of the Revised Faces Pain Scale and of the Barthel Index at all moments of the study. A decrease in the pain scores can be noted in both scales that measure this symptom, with this improvement being continuous and progressive in the FPS-R, reaching its peak at six months post-operative. The results of the Barthel Index, an instrument used to assess the performance of activities of daily living (ADLs), show an improvement of approximately $10 \%$ at $\mathrm{T} 1$ and $\mathrm{T} 2$. At six months, there is an increase in the mean of this index, reaching almost $30 \%$ gain in the ADLs of the patients. 
Table 3 - Descriptive analysis of the pain variable of the EORTC QLQ-C30 instrument, the revised Faces Pain Scale (FPS-R) and the Barthel index of the patients with vertebral metastasis of the Clinical Hospital of Ribeirão Preto Medical School (HC -FMRP), Ribeirão Preto/SP - Brazil, 2013-2015 (n = 22).

\begin{tabular}{|l|c|c|c|c|c|}
\hline \multicolumn{1}{|c|}{ Variable } & Minimum value & Maximum value & Mean & Median & SD \\
\hline Pain at $T 0$ & 33 & 100 & 83.33 & 83.33 & 18.54 \\
\hline Pain at $T 1$ & 0 & 100 & 35.29 & 33.33 & 32.74 \\
\hline Pain at $T 2$ & 0 & 100 & 45.24 & 33.33 & 36.06 \\
\hline Pain at T3 & 17 & 100 & 44.44 & 33.33 & 26.90 \\
\hline FPS- $\boldsymbol{R}^{*}$ at $T 0$ & 2 & 10 & 6.18 & 6.00 & 2.54 \\
\hline FPS-R at $T 1$ & 0 & 6 & 3.18 & 4.00 & 2.45 \\
\hline FPS-R at T2 & 0 & 6 & 3.00 & 2.00 & 2.18 \\
\hline FPS-R at T3 & 0 & 6 & 2.17 & 2.00 & 1.80 \\
\hline Barthel at T0 & 20 & 100 & 61.59 & 67.50 & 26.61 \\
\hline Barthel at $T 1$ & 5 & 100 & 68.82 & 80.00 & 32.24 \\
\hline Barthel at T2 & 0 & 100 & 67.50 & 87.50 & 37.56 \\
\hline Barthel at T3 & 25 & 100 & 79.58 & 95.00 & 28.96 \\
\hline
\end{tabular}

* Revised Faces Pain Scale

\section{Discussion}

Surgical treatment of spinal decompression for patients with vertebral metastasis involves a delicate balance between survival, functionality and health related quality of life. Achieving better control of symptoms and a satisfactory level of functionality, can enable these patients to return to their homes and the social lives they once had. Furthermore, the percentage of these patients that are institutionalized and/or hospitalized can be decreased.

The sociodemographic characterization of the patients with vertebral metastases that composed the study sample showed an equal distribution of men and women, with a little more than half of the participants being aged less than 60 years. In the literature a high incidence of vertebral metastases was found in individuals of 40 to 65 years of age, which is the period of greatest risk of developing cancer, particularly breast cancer, which is the most prevalent in females, except for the non-melanoma skin cancers [17-18]. If all age groups are considered, an increase in the prevalence of cases of vertebral metastasis can be seen in men, because after 65 years there is a significant increase in cases of prostate cancer, and this, in absolute numbers, has a higher prevalence when compared to breast cancer in women [18-19]. According to the biannual estimate made by the National Cancer Institute of Brazil (INCA) for the biennium 2014-2015, the number of new cases of prostate cancer is 69,000/year and breast cancer 57,000/year [20].

Despite a higher expectation of metastasis diagnosis in the male population due to a higher incidence of prostate cancer, in the present study the prevalence was equal among men and women. In the sample of 22 patients, nine patients had a diagnosis of breast cancer $(40.9 \%)$, explaining the proportional increase in cases among women. Regarding the prevalence of primary tumor sites, breast cancer was followed by prostate cancer (18.2\%), lung cancer (13.6\%) and colorectal cancer (13.6\%). Similar epidemiological data has also been seen in other published studies [21-22]. A probable explanation for the reversal of the prevalence of metastases in the spine between prostate cancer and breast cancer may be due to the indolence of the evolution of the metastatic status of prostate cancer. This tumor can be controlled by hormonal manipulation in order to reduce testosterone production (orchiectomy or use of luteinizing hormone-releasing hormone receptor agonists) or through radiotherapy to control bone metastasis prior to spinal surgery [23].

From the oncologic perspective, metastatic spinal cord compression generates symptoms such as pain, neurological deficits and bladder or bowel incontinence. These symptoms significantly reduce the quality of life of the patient in these situations and clinical treatment is usually ineffective. Although surgery for metastatic spinal decompression is considered palliative, it can provide potential benefits to reduce pain or prevent the progression of a neurological deficit of the patient. Achieving some of these objectives, it is possible to attribute an improvement in the quality of life of these individuals.

The older surgical techniques of spinal decompression without spinal stabilization presented poor results. Thus, in the past, radiotherapy was the treatment modality most frequently used for management of the pain of vertebral metastasis. More recent evidence has shown that the current surgical methods, including anterior and posterior access with spinal stabilization, provide better results compared to radiotherapy as a single method of treatment [24].

The choice of each approach depends on the location of the tumor, the type of reconstruction required, the comorbidities presented by the patients and the extent of the disease in the vertebral segment affected. The most widely used procedure in this study was the posterior vertebral approach, occurring in over $60 \%$ of cases. This choice correlates with the higher prevalence of involvement of the thoracic and lumbar levels, which together accounted for nearly $82 \%$ of the lesions. Several studies in the literature show that posterior access is the most appropriate for these two vertebral levels [5,25]. 
The treatment offered to patients with vertebral metastasis is intended to improve the symptoms that impair the performance of their daily functions, directly interfering with their Quality of Life. A Canadian study [26] evaluated the impact of surgery on the Quality of Life of patients with vertebral metastases up to one year after surgery. The author used the same Health Related Quality of Life instrument (EORTC QLQ-C30) as this study and found significant differences only for the Global Health Status/Quality of Life (GHS/QoL) Scale ( $p=$ $0.013)$ and Pain $(p=0.0016)$ up to the sixth month. The functional capacity of these patients was evaluated through the physical performance scale ECOG (Eastern Cooperative Oncology Group) Performance Status. The analysis of this variable in relation to the surgical procedure showed no significant gains. These results confirm the central role of pain in the evaluation of the Global Quality of Life of metastatic cancer patients.

A French study [27] also evaluated a population of patients in the same clinical conditions and found similar results. For the GHS/QoL and Pain items, there was a significant improvement comparing the preoperative moment with the other post-operative moments evaluated (one, three, six and twelve months) ( $p$ $<0.0001)$. Functional capacity was assessed using the Frankel scale, widely used in spinal cord injury. This scale evaluates the level of bone marrow involvement, the degree of remaining muscle strength and the dermatome affected. It is classified into A, B, C, D or E, where classification A corresponds to complete injury with both motor and sensory involvement below the level of injury and classification $\mathrm{E}$ to no neurological deficit with preserved motor and sensory functions. The post-surgery results showed that almost $100 \%$ of the patients showed improvement or stabilization of the Frankel scale evaluation when compared to the pre-operative results.

In a Brazilian [28] study, which also evaluated the Quality of Life of individuals before and after spinal decompression procedure caused by metastasis (pre-operatively, one and six months), the SF-36 (Short-Form Health Survey) was used. The results were statistically significant for the Pain $(\mathrm{p}=0.004)$, Functional Capabilities ( $\mathrm{p}<0.001)$, Social Aspects ( $\mathrm{p}<0.001)$ and Mental Health ( $\mathrm{p}<0.001)$ items, for both the first month after surgery, as well as for the sixth month.

Based on the literature mentioned we can say that the findings of the present study were similar to those previously described. The instrument used to evaluate the Health Related Quality of Life in the population with cancer enabled us to clearly visualize the results presented by them. With pain being the main symptom reported by individuals at T0, this showed significant improvement at T1, T2 and T3. In order to better understand the physical limitations experienced on a daily basis, the activities of daily living were evaluate through the Barthel Index, which showed no statistical difference at any moment of the study. This index was not used in any other study in the literature cited above, however, functional performance was evaluated in all with other scales. Even using various scales, the statistical results were generally unsatisfactory.

The importance of these results is clear, especially regarding the improvements related to the pain reported by the patients and the avoidance of an irreversible neurological impairment that would also impact the quality of life. The limitations of this study that should be mentioned include the lack of a control group, as withholding treatment or simulating surgery could not be ethically proposed. There was also a significant loss in the follow-up due in part to the morbidity and mortality associated with cancer in general or even the inherent psychosocial problems of the disease in question.

\section{Conclusion}

The results of this study showed that surgical intervention in cases of spinal cord compression caused by metastasis provides an improvement in pain control and, as a result, provides a gain in Health Related Quality of Life for these patients.

\section{References}

[1]. A.J. Aboulafia, A.M. Levine, D. Schmidt, and Aboulafia D. Surgical therapy of bone metastases. Seminars in Oncology, 34, 2007, 206-214.

[2]. R. Wedin, H.C. Bauer, and L.E. Rutqvist. Surgical treatment for skeletal breast cancer metastases: a population-based study of 641 patients. Cancer, 92, 2001, 257-262.

[3]. F. Zairi, A. Arikat, M. Allaoui, P. Marinho, and R. Assaker. Minimally invasive decompression and stabilization for the management of thoracolumbar spine metastasis. J Neurosurg Spine, 17, 2012, 19-23.

[4]. G. Maccauro, M.S. Spinelli, S. Mauro, C. Perisano, C. Graci, and M. A. Rosa. Physiopathology of spine metastasis. Int J Surg Oncol, 2011, 2011, 1-8.

[5]. T.F. Witham, Y.A. Khavkin, G.L. Gallia, J.P. Wolinsky, and Z.L. Gokaslan. Surgery insight: current management of epidural spinal cord compression from metastatic spine disease. Nat Clin Pract Neurol, 2, 2006, 87-94.

[6]. S.A. Meyer, H. Singh, and A.L. Jenkins. Surgical treatment of metastatic spinal tumors. Mt Sinai J Med, 77, 2010, 124-129.

[7]. R.L. Ruff, S.S. Ruff, and X. Wang. Persistent benefits of rehabilitation on pain and lifequality for nonambulatory patients with spinal epidural metastasis. J Rehabil Res Dev, 44, 2007, 271-278.

[8]. R.W. Gilbert, J.H. Kim, and J.B. Posner. Epidural spinal cord compression from metastatic tumor: diagnosis and treatment. Ann Neurol, 3, 1978, 40-51.

[9]. A.D. Aaron. Current concepts review-treatment of metastatic adenocarcinoma of the pelvis and the extremities. J Bone Joint Surg, 79, 1997, 917-32. 
[10]. W.D. Hage, A.J. Aboulafia, and D.M. Aboulafia. Incidence, location, and diagnostic evaluation of metastatic bone disease. Orthop Clin North Am, 31, 2000, 515-28

[11]. J.C. Wang, P. Boland, N. Mitra, Y. Yamada, E. Lis, M. Stubblefield, et al. Single-stage posterolateral transpedicular approach for resection of epidural metastatic spine tumors involving the vertebral body with circumferential reconstruction: results in 140 patients. Invited submission from the Joint Section Meeting on Disorders of the Spine and Peripheral Nerves, March 2004. $J$ Neurosurg Spine, 1, 2004, 287-98.

[12]. E.K. Wai, J.A. Filkestein, R.P. Tangente, L. Holden, E. Chow, M. Ford, et al. Quality of life in surgical treatment of metastatic spine disease. Spine, 28, 2003, 508-12.

[13]. M.M. Shannon, M.A. Ryan, N. D’Agostino, and F.J. Brescia. Assessment of pain in advanced cancer patients. J Pain Symptom Manage, 10, 1995, 274-8.

[14]. N.K. Aaronson, S. Ahmedzai, B. Bergman, M. Bullinger, A. Cull, N.J. Duez, et al. The European Organization for Research and Treatment of Cancer QLQ-C30: a quality-of-life instrument for use in international clinical trials in oncology. J Natl Cancer Inst, 85, 1993, 365-76.

[15]. P.M. Fayers. Interpreting quality of life data: population-based reference data for the EORTC QLQ-C30. Eur J Cancer, 3, 2001, 1331-4.

[16]. F.I. Mahoney, and D.W. Barthel. Functional evaluation: the Barthel Index. Md State Med J, 14, 1965, 61-65

[17]. R.J. Petteys, D.M. Sciubba, and Z.L. Gokaslan. Surgical management of metastatic spine disease. Seminars in Spine Surgery, 21, 2009, 86-92

[18]. D.M. Sciubba, R.J. Petteys, M.B. Dekutoski, C.G. Fisher, M.G. Fehlings, S.L. Ondra, et al. Diagnosis and management of metastatic spine disease. J Neurosurg Spine, 13, 2010, 94-108.

[19]. R.G. Perrin, and A.W. Laxton. Metastatic spine disease: epidemiology, pathophysiology and evaluation of patients. Neurosurg Clin $N$ Am, 15, 2004, 365-73

[20]. Instituto Nacional do Câncer (INCA). Estimativa 2014: incidência de câncer no Brasil. Coordenação de Prevenção e Vigilância. Rio de Janeiro, 2014; [citado em 2015 May 14]. Disponível em: http://www.inca.gov.br/estimativa/2014. Citado em 08/11/2016.

[21]. W.A. Abdu, and M. Provencher. Primary bone and metastatic tumors of the cervical spine. Spine (Phila Pa 1976), 23, 1998, 276777.

[22]. A. Solberg, and R.M. Bremnes. Metatatic spinal cord compression: diagnostic delay, treatment, and outcome. Anticancer Res, 19, $1999,677-84$

[23]. J. Seidenfeld, D.J. Samson, V. Hasselblad, N. Aronson, P.C. Albertsen, C.L. Bennett, et al. Single-therapy androgen suppression in men with advanced prostate cancer: a systematic review and meta-analysis. Ann Inter Med, 132, 2000, 566-77.

[24]. D. Choi, A. Crockard, C. Bunger, J. Harms, N. Kawahara, C. Mazel, et al. Review of metastatic spine tumor classification and indications for surgery: the consensus statement of the Global Spine Tumor Study Group. Eur Spine, 19, 2010, 215-22.

[25]. M.P. Steinmetz, A. Mekhail, and E.C. Benzel. Management of metastatic tumors of the spine: strategies and operative indications. Neurosurg Focus, 11, 2001, e2.

[26]. A. Falicov, C.G. Fisher, J. Sparkes, M.C. Boyd, P.C. Wing, and M.F. Dvorak. Impact of surgical intervention on quality of life in patients with spinal metastases. Spine (Phila Pa 1976), 31, 2006, 2849-56.

[27]. G.M. Quan, J.M. Vital, N. Aurouer, I. Obeid, J. Palussière, A. Diallo, et al. Surgery improves pain, function and quality of life in patients with spinal metastases: a prospective study on 118 patients. Eur Spine J, 20, 2011, 1970-8.

[28]. L.M.R. Rodrigues, E.S. Valesin Filho, F.H. Ueno, E.M. Fujiki, and C. Milani. Qualidade de vida de pacientes submetidos à descompressão por lesão vertebral metastática. Acta Ortop Bras, 19, 2011, 149-53 